ECCOMAS

Proceedia
UNCECOMP 2017

$2^{\text {nd }}$ ECCOMAS Thematic Conference on Uncertainty Quantification in Computational Sciences and Engineering M. Papadrakakis, V. Papadopoulos, G. Stefanou (eds.) Rhodes Island, Greece, 15-17 June 2017

\title{
REDUCED ORDER MODEL-BASED UNCERTAINTY MODELING OF STRUCTURES WITH LOCALIZED RESPONSE
}

\author{
Pengchao Song and Marc P. Mignolet \\ SEMTE Faculties of Mechanical and Aerospace Engineering \\ Arizona State University, Tempe, AZ 85287, USA \\ e-mail: pengchao.song@asu.edu, marc.mignolet@asu.edu
}

Keywords: Uncertainty Modeling, Reduced Order Modeling, Maximum Entropy, Structural Uncertainty, Localized Response.

\begin{abstract}
This paper focuses on the introduction of uncertainty in reduced order models of structures exhibiting a localized static response in the neighborhood of the excitation. A straightforward application of the maximum entropy framework is first considered to carry out the stochastic modeling of the uncertainty. Quite consistently with the maximization of the entropy, it is found that this modeling may lead to a "globalization" of the response and thus an extension of the nonparametric stochastic modeling approach is sought. To this end, the eigenvalues and eigenvectors of the stiffness matrix of structures exhibiting this localization property are first studied. It is found that their lowest eigenvalues are closely spaced when the corresponding eigenvectors are extended to the entire structure. On this basis, a novel version of the nonparametric stochastic modeling approach is introduced to randomize the entire stiffness matrix while distorting only slightly the closely spaced eigenvalue structure. The above concepts are demonstrated on a thin annulus clamped at its inner radius and a localization of the uncertain response is indeed observed using the proposed approach.
\end{abstract}

(C)2017 The Authors. Published by Eccomas Proceedia.

Peer-review under responsibility of the organizing committee of UNCECOMP 2017.

doi: $10.7712 / 120217.5389 .17224$ 


\section{INTRODUCTION}

In the last two decades or so, significant progress has been achieved toward the rational consideration of uncertainty in engineering systems to better predict their performance and the variability thereof. Among the techniques developed within this time frame is the maximum entropy-based nonparametric approach which was initially proposed [1] within the context of modal models of structures. It is however applicable much more broadly (e.g., see [2] for a review) especially when projection based reduced order models are employed. Such models are traditionally developed for computational reasons, being much faster than their full order counterparts, e.g., finite element models. However, from the standpoint of uncertainty modeling, they have yet another advantage as they "concentrate" the uncertainty originating from multiple sources into the single set of their parameters which are typically matrices. The reduced order modeling framework thus permits the consideration of uncertainty in well defined parameters of the physical systems, e.g., thickness, density, etc., but also of harder to model uncertainties such as geometry, material constitutive behavior, etc., as long as they do not affect the form of the reduced order model.

Since its initial formulation in [1], the maximum entropy-based nonparametric approach has been extended multiple times to cover new classes of problems, e.g., vibro-acoustics [3,4], rotordynamics [5-7], nonlinear structural dynamics [8,9], nonlinear thermoelastic problems [10], linear viscoelastic structures [11], etc., but also in rigid body dynamics $[12,13]$ and micromechanics and multiscale modeling, see [2]. The focus of the present effort is on yet another extension of this approach, more specifically to problems in which (i) the response of the mean model (i.e., the one without uncertainty) is localized to one part of the spatial domain and (ii) the uncertainty to be modeled does not alter this localization. The static response of some structures does satisfy the above conditions, and one such example described next will be used as application. There are however other non-structural applications in which these conditions are encountered, e.g., in some heat conduction problems (see [14]), and to which the present discussion may also apply.

\section{REPRESENTATIVE EXAMPLE}

To illustrate the class of problems investigated here, consider the annulus shown in Fig. 1(a) of inner radius $0.8 \mathrm{~m}$, outer radius $1 \mathrm{~m}$, thickness $0.002 \mathrm{~m}$ clamped on its inner radius and free on the outer one. The material, aluminum, is assumed to be homogenous and isotropic with Young's modulus $E=7.310^{10} \mathrm{~Pa}$ and Poisson's ratio $v=0.316$. The annulus is subjected to a static uniform unit pressure in the quadrant $\theta \in[180,270]$ degrees highlighted in yellow in Fig. 1(a). To evaluate the displacement field of the annulus, it was modeled by finite elements within Nastran (CQUAD4 elements) with a mesh of 144 nodes around the periphery and 6 in the radial direction. Then, shown in Fig. 1(b) is the transverse displacement of the periphery which is clearly localized near the excitation, i.e., in the band $\theta \in[150,300]$ degrees.

A reduced order model of the finite element one can be constructed by representing the nodal responses stacked in the vector $\underline{u}$ as a linear combination of basis functions $\underline{\psi}_{i}$, i.e. as

$$
\underline{u}=\sum_{i=1}^{M} q_{i} \underline{\psi}_{i}
$$

where the variables $q_{i}$ are referred to as generalized coordinates. The adoption of the representation of Eq. (1) transforms the equations for the nodal displacements

$$
\bar{K}_{F E} \underline{u}=\bar{F}_{F E},
$$

where $\bar{K}_{F E}$ and $\underline{\bar{F}}_{F E}$ are the finite element stiffness matrix and loading, into 


$$
\bar{K} \underline{q}=\underline{F}
$$

where

$$
\bar{K}=\Psi^{T} \bar{K}_{F E} \Psi \quad \text { and } \quad \underline{\bar{F}}=\Psi^{T} \underline{\bar{F}}_{F E}
$$

with ${ }^{T}$ denoting the operation of matrix transposition and $\Psi=\left[\underline{\Psi}_{1} \underline{\Psi}_{2} \underline{\Psi}_{3} \cdots \underline{\Psi}_{M}\right]$.

The above reduced order model construction was exemplified by selecting the basis functions $\underline{\Psi}_{i}$ as the linear modes of the annulus. Then, shown in Fig. 1(b) is the transverse displacement of the periphery obtained with 55 such modes that, as expected, closely approximates the finite element solution.

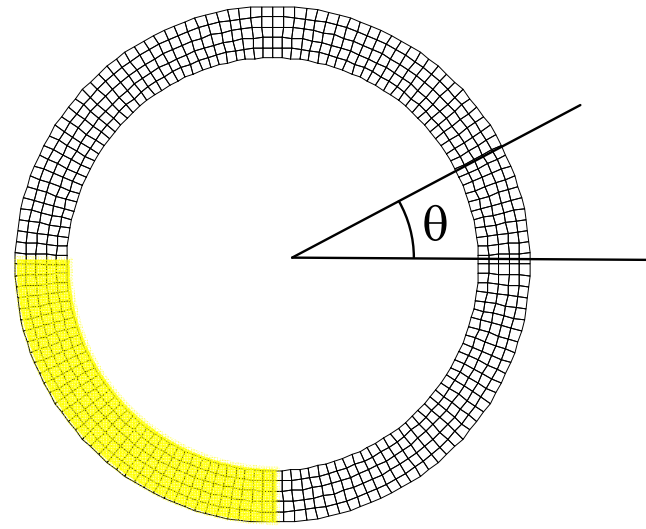

(a)

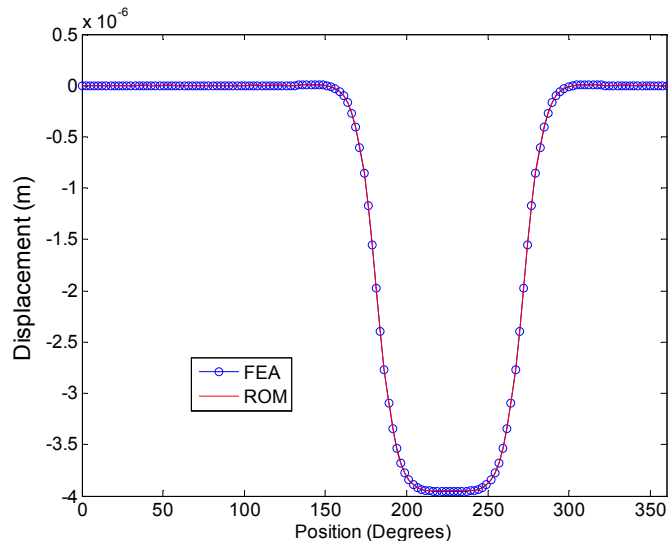

(b)

Figure 1: (a) The annulus and its finite element model with the loading domain highlighted in yellow. (b) Static transverse displacements at the periphery, full finite element (FEA) and reduced order (ROM) models.

\section{STOCHASTIC UNCERTAINTY MODELING}

\section{1 "Standard" Maximum Entropy Nonparametric Approach}

The consideration of uncertainty in the properties and/or geometry of the structural model can be carried out on the finite element model by letting a set of its parameters, e.g., Young's modulus, Poisson's ratio, thickness, outer/inner radii, etc. be random variables with known joint probability density function. Then, proceeding with a Monte Carlo simulation of these properties and performing a finite element analysis for each sample will provide a population of responses which can be analyzed to assess the effects of uncertainty. This approach is appropriate, although potentially computationally expensive, to address the uncertainty in $p a$ rameters of the model such as those stated above. It is however much more difficult to implement when the uncertainty originates from waviness in the periphery, deviations from the isotropic and homogenous material properties, etc.

While the finite element model will vary, the form of the equations for the response, i.e., Eq. (2), will remain unchanged as long as the constitutive model is linear and that the deformations remain small. Similarly, Eq. (3) will also remain valid as long as the basis functions, assumed here to be deterministic, provide an appropriately accurate representation of the random displacement field. This observation suggests the possibility of introducing the uncertainty directly in either Eq. (2) or Eq. (3). In fact, proceeding with Eq. (3) is easier as (i) $\bar{K}$ is 
a typically much smaller matrix than $\bar{K}_{F E}$ and (ii) the former does not exhibit a particular topology as opposed to the latter.

Having established the desire to introduce the uncertainty directly in the reduced order model stiffness matrix $\bar{K}$, it remains to address how to proceed and more specifically how to select the joint probability density function of the elements of this matrix. The framework selected here is the maximum entropy-based nonparametric approach proposed by Soize [1], see [2] for an extensive review. Within this framework, the joint probability density function of the elements of the stiffness matrix $\bar{K}$ is not selected but rather determined to maximize the corresponding entropy under constraints corresponding to all physical properties it must satisfy.

More specifically, it is known that the finite element stiffness matrix $\bar{K}_{F E}$ must be symmetric and positive definite (strictly positive definite given the clamp boundary conditions) and those properties are transferred to its reduced order model counterpart $\bar{K}$ through the transformation of Eq. (4). Then, proceeding as in [1], see also [2], this stiffness matrix of the "mean model", i.e., the model without uncertainty, is first decomposed as

$$
\bar{K}=\bar{L} \bar{L}^{T}
$$

e.g., by Cholesky factorization. Then, random matrices $K$ are generated as

$$
K=\bar{L} H H^{T} \bar{L}^{T}
$$

where $H$ is a lower triangular matrix such that (see also Fig. 2)

(1) its off-diagonal elements $H_{i l}, i \neq l$, are normally distributed (Gaussian) random variables with standard deviation $\sigma=1 / \sqrt{2 \mu}$, and

(2) its diagonal elements $H_{i i}$ are obtained as $H_{i i}=\sqrt{Y_{i i} / \mu}$ where $Y_{i i}$ is Gamma distributed with parameter $(p(i)-1) / 2$ where

$$
p(i)=n-i+2 \lambda_{0}-1 \text { and } \mu=\left(n+2 \lambda_{0}-1\right) / 2
$$

In the above equations, $n$ is the size of the matrices and the parameter $\lambda_{0}>0$ is the free parameter of the statistical distribution of the random matrices $K$. An alternative parametrization is through the dispersion parameter $\delta$ defined as

$$
\delta^{2}=\frac{n+1}{n+2 \lambda_{0}-1} .
$$

zero mean Gaussian, independent of each other with standard dev. $\sigma=1 / \sqrt{2 \mu}$

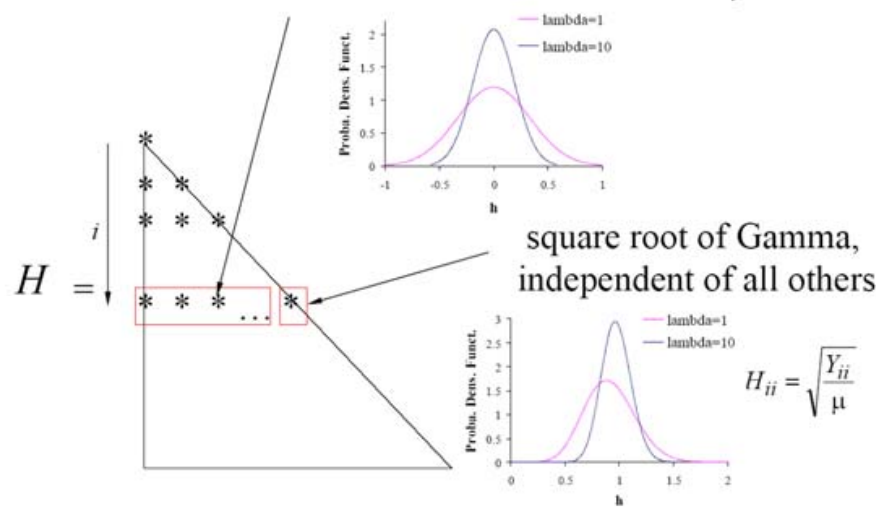

Figure 2: Structure of the random $H$ matrices (figures for $n=8, i=2$, and $\lambda=1$ and 10). 


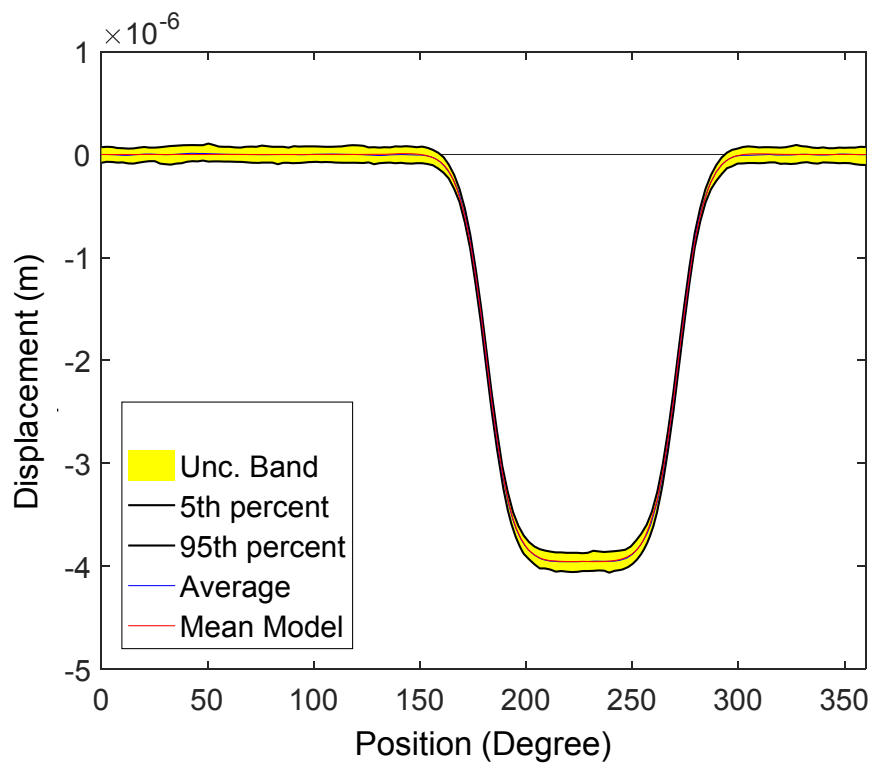

Figure 3: Static transverse displacement at the periphery of the mean annulus (in red) and 5th-95th percentile uncertainty band (in yellow) of the displacement, standard stochastic reduced order model.

The above approach was applied as is to the $55 \times 55$ stiffness matrix of the reduced order model of the annulus and 300 random matrices $K$ were determined. From each one of those, a set of generalized coordinates $q$ was determined that satisfies

$$
K \underline{q}=\underline{F}
$$

and the resulting set of nodal displacements $\underline{u}$ were obtained from Eq. (1). Shown in yellow in Fig. 3 is the uncertainty band corresponding to the 5th and 95th percentile of the transverse displacement of the periphery as determined from the 300 samples of the response. Comparing Figs 1(b) and 3, it is observed that the above uncertainty modeling approach has induced some globalization of the response, i.e., the response outside of the region $\theta \in[150,300]$ degrees is not nearly zero for all samples as could have been construed from Fig. 1(b). Note that this effect likely leads to a higher entropy of the response as compared to the localized case given its increased spread of the joint probability density function of this response. This finding seems quite consistent with, although separate from, the maximization of entropy of the matrix $K$ which is guaranteed by the construction of Eqs (6)-(8).

It should be recognized that the above globalization effect is certainly physically possible, e.g., it could take place if the disk on which the annulus is clamped is not rigid as specified in the mean model but rather exhibits some flexibility which induces a long range interaction between nodal responses.

If, however, there is additional knowledge about the uncertainty that indicates that such a globalization does not take place, then the above methodology must be modified to reflect it, e.g., by adding constraints in the optimization of the entropy or modeling differently the random stiffness matrix. One such extension is formulated below.

\subsection{Localization Inducing Property}

Before revising the above standard nonparametric approach, it is necessary to identify the property of the stiffness matrices $\bar{K}$ and $\bar{K}_{F E}$ that induces the existence of a localized response. To this end, recall that the solution $\underline{u}$ of Eq. (1) can be expanded in terms of the ei- 
genvectors $\underline{\phi}_{j}$ and corresponding eigenvalues $\lambda_{j}$ of $\bar{K}_{F E}$ as

$$
\underline{u}=\sum_{j} \frac{\left(\underline{\phi}_{j}^{T} \bar{F}_{F E}\right)}{\lambda_{j}} \underline{\phi}_{j}
$$

owing to the orthogonality of the eigenvectors implied by the symmetry of $\bar{K}_{F E}$.

To proceed further in the discussion, assume first that the eigenvectors $\underline{\phi}_{j}$ are extended to the entire structure; this is true of the annulus of Fig. 1(a) for which $\underline{\phi}_{j}$ are harmonic functions of the angle $\theta$ (as discretized by the finite element modeling). Then:

(i) a localized response as shown in Fig. 1(b) is possible only if the dominant coefficients $\left(\underline{\phi}_{j}^{T} \bar{F}\right) / \lambda_{j}$ have somewhat similar values as to create an appropriate mix of the extended functions $\underline{\phi}_{j}$ to produce localization.

(ii) the "modal forces" $\underline{\phi}_{j}^{T} \overline{\underline{F}}$ decrease slowly as the index $j$ is increased given the localization of the excitation or equivalently the values $\underline{\phi}_{j}^{T} \overline{\underline{F}}$ for the dominant modes will be quite similar.

Combining these two arguments, it is thus concluded that localization must require that the lowest eigenvalue $\lambda_{j}$ of $\bar{K}_{F E}$ be close together if the eigenvectors of this stiffness matrix are extended. This result is confirmed by the plot of eigenvalues of the annulus' stiffness matrix shown in Fig. 4: the first few of its eigenvalues are indeed closely spaced.

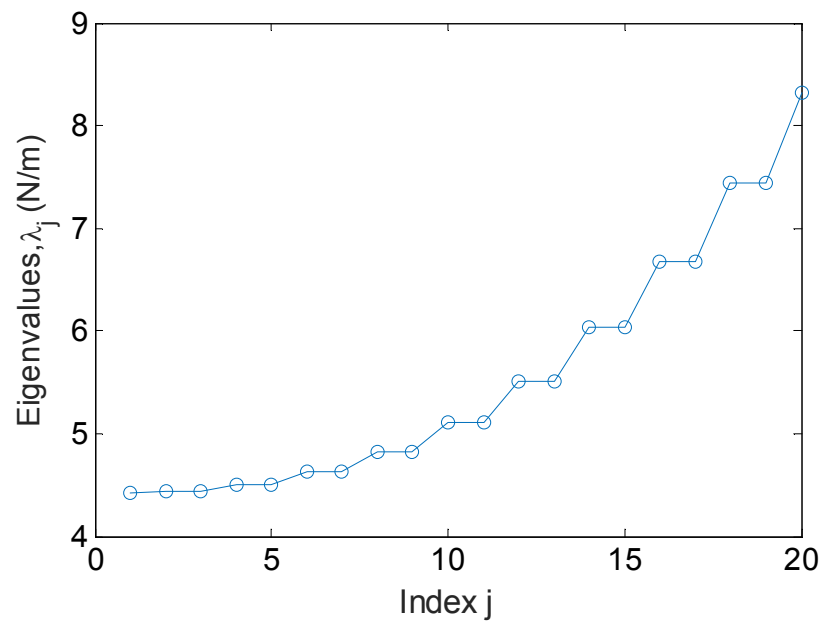

Figure 4. Eigenvalues of the stiffness matrix of the annulus finite element increasing order.

For structures with localized response and well separated eigenvalues of their stiffness matrix, the change in stiffness matrix induced by the random matrix $H$ (Eq. (6)) produces only small changes in eigenvalues and eigenvectors when the uncertainty is small (see [15] for perturbation analysis) and thus small changes in the response. That is, for these structures, the application of the standard nonparametric approach may maintain the localized behavior at least as long as the change in eigenvalues and eigenvectors induced by the uncertainty remains small enough. 
The above comments suggest that what is important for localization is the relative separation of the first few eigenvalues which must be small when the eigenvectors are extended. To confirm this statement, rewrite Eq. (10) in the form

$$
\underline{u}=\frac{1}{\lambda_{1}} \sum_{j}\left(\underline{\phi}_{j}^{T} \bar{F}_{F E}\right) \underline{\phi}_{j}+\frac{1}{\lambda_{1}} \sum_{j} \frac{\left(\lambda_{1}-\lambda_{j}\right)}{\lambda_{j}}\left(\underline{\phi}_{j}^{T} \bar{F}_{F E}\right) \underline{\phi}_{j} .
$$

or

$$
\underline{u}=\left[\frac{1}{\lambda_{1}} \sum_{j} \underline{\phi}_{j} \underline{\phi}_{j}^{T}\right] \overline{\bar{F}}_{F E}+\left[\frac{1}{\lambda_{1}} \sum_{j} \frac{\left(\lambda_{1}-\lambda_{j}\right)}{\lambda_{j}} \underline{\phi}_{j} \underline{\phi}_{j}^{T}\right] \underline{\bar{F}}_{F E}=\bar{P}_{1} \underline{\bar{F}}_{F E}+\bar{P}_{2} \underline{\bar{F}}_{F E} .
$$

where $\bar{P}_{1}$ is proportional to the identity matrix and thus $\bar{P}_{1} \underline{\bar{F}}$ is a purely local term, i.e., the corresponding displacement at each node only depends on the force acting at that node. This term will dominate when the terms $\left(\underline{\phi}_{j}^{T} \bar{F}\right)\left(\lambda_{1}-\lambda_{j}\right) / \lambda_{j}$ are all small, i.e., when the dominant eigenvectors (those with significant values of $\underline{\phi}_{j}^{T} \overline{\underline{F}}$ ) have a small relative difference of eigenvalue with eigenvector 1 .

A similar discussion to the above one applies for the mean reduced order stiffness matrix $\bar{K}$ of eigenvectors $\underline{\varphi}_{j}$ and eigenvalues $\mu_{j}$ so that

$$
\underline{q}=\bar{Q} \underline{\bar{F}}=\left[\frac{1}{\mu_{1}} \sum_{j} \underline{\varphi}_{j} \underline{\varphi}_{j}^{T}\right] \overline{\bar{F}}+\left[\frac{1}{\mu_{1}} \sum_{j} \frac{\left(\mu_{1}-\mu_{j}\right)}{\mu_{j}} \underline{\varphi}_{j} \underline{\varphi}_{j}^{T}\right] \overline{\underline{F}}=\bar{Q}_{1} \overline{\bar{F}}+\bar{Q}_{2} \underline{\bar{F}} .
$$

Associated to this decomposition of the flexibility matrix $\bar{Q}$ into a component inducing local effects $\left(\bar{Q}_{1}\right)$ and a more global one $\left(\bar{Q}_{2}\right)$ is a similar split of the stiffness matrix $\bar{K}$ into a local component, $\bar{K}_{L}$, and a more global one, $\bar{K}_{G}$, defined as

$$
\bar{K}_{L}=\left[\bar{Q}_{1}\right]^{-1}=\mu_{1} \sum_{j} \underline{\varphi}_{j} \underline{\varphi}_{j}^{T} \quad \text { and } \quad \bar{K}_{G}=\bar{K}-\bar{K}_{L} .
$$

\subsection{Maximum Entropy Nonparametric Modeling for Localized Responses}

The above discussion has demonstrated that a localization of the response will only occur when the relative spread of the dominant eigenvalues of the stiffness matrix is small when the corresponding eigenvectors are extended. However, this spread is often increased, sometimes very significantly for the first few eigenvalues, when using the standard nonparametric approach as shown in Fig. 5.

Thus, imposing a localization constraint on the stochastic modeling will require controlling the eigenvalues of the dominant modes (those with eigenvalues close to $\lambda_{1}$ ) separately from the rest of them. In this regard, note that the dominant eigenvectors are mostly present in $\bar{K}_{L}$ while those with eigenvalues far from $\lambda_{1}$ are dominant in $\bar{K}_{G}$.

On the basis of the above observations, it is proposed here to model the uncertainty in $\bar{K}_{L}$ and $\bar{K}_{G}$ separately. Since there is no particular requirement on the latter matrix (which is nevertheless symmetric and positive definite) and its randomization, the standard nonparametric approach will be applied leading to an uncertain matrix $K_{G}$ defined as

$$
K_{G}=\bar{L}_{G} H_{G} H_{G}^{T} \bar{L}_{G}^{T} \quad \text { where } \quad \bar{K}_{G}=\bar{L}_{G} \bar{L}_{G}^{T}
$$




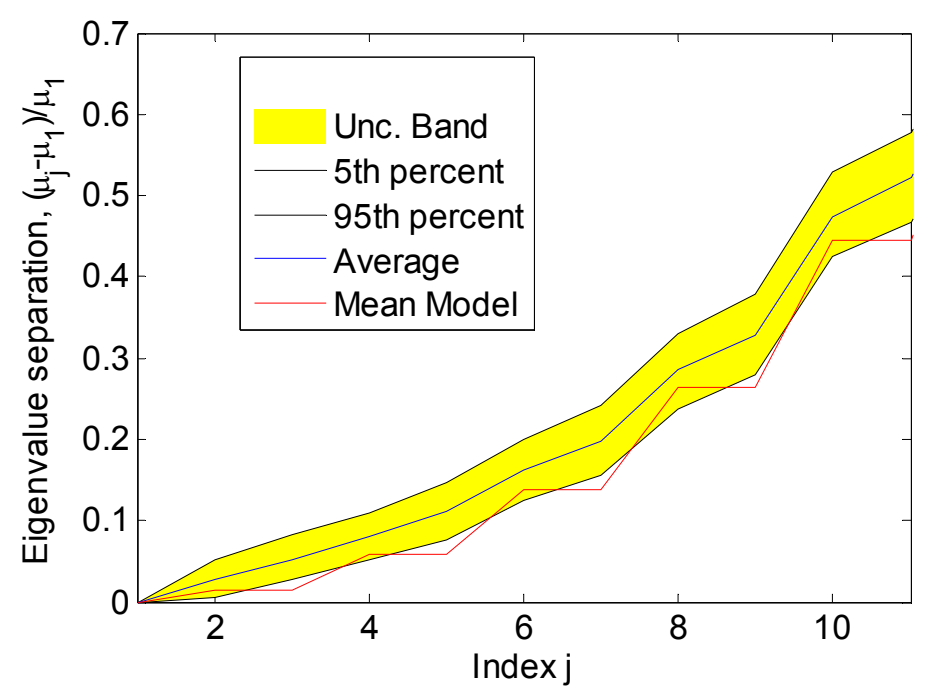

Figure 5: Relative eigenvalue separation of the reduced order model stiffness matrix. Mean model (red) and 5th-95th percentile uncertainty band, standard stochastic model.

with $H_{G}$ a lower triangular random matrix as defined by Eqs (7)-(8) and Fig. 2 for a particular dispersion $\delta_{G}$.

Maintaining small the relative separation between the eigenvalues of the dominant eigenvectors can be achieved by scaling uniformly all eigenvalues. This observation suggests that a first approach to introduce uncertainty in $\bar{K}_{L}$ is to simply multiply it by a random variable $\Lambda$ which consistently with the maximum entropy concepts can be selected as $H_{1}^{2}$ where $H_{1}$ is a $1 \mathrm{x} 1$ matrix defined as in Eqs (7)-(8) and Fig. 2 with a specified dispersion $\delta_{1}$. A small change in the relative separation between eigenvalues can also be induced by splitting $\bar{K}_{L}$ as $\bar{K}_{G}$ in Eq. (15) with a random lower triangular matrix $H_{L}$ of dispersion $\delta_{L}$. Combining the above two operations leads to the proposed model

$$
K_{L}=H_{1}^{2} \bar{L}_{L} H_{L} H_{L}^{T} \bar{L}_{L}^{T} \quad \text { where } \quad \bar{K}_{L}=\bar{L}_{L} \bar{L}_{L}^{T} .
$$

Note finally that since $\bar{K}_{L}$ is defined by Eq. (14a), its decomposition in Eq. (16b) is readily achieved by selecting

$$
\bar{L}_{L}=\sqrt{\mu_{1}}\left[\varphi_{1} \underline{\varphi}_{2} \underline{\varphi}_{3} \cdots\right]
$$

The uncertain reduced order stiffness matrix is then obtained as

$$
K=K_{L}+K_{G} \text {. }
$$

\subsection{Application to the Annulus}

The three-parameter $\left(\delta_{G}, \delta_{1}, \delta_{L}\right)$ stochastic model formulated above was applied to the reduced order model of the annulus with the first 8 eigenvectors $\underline{\varphi}_{j}$ retained in the summation of Eq. (14a). To highlight the effects of each random component on the response, shown in Figs 6 (a),(c),(e) are the uncertainty bands obtained with each of the parameter set to a nonzero value in turn. Also shown, on Figs 6 (b),(d),(f) are 3 samples of the corresponding responses. 

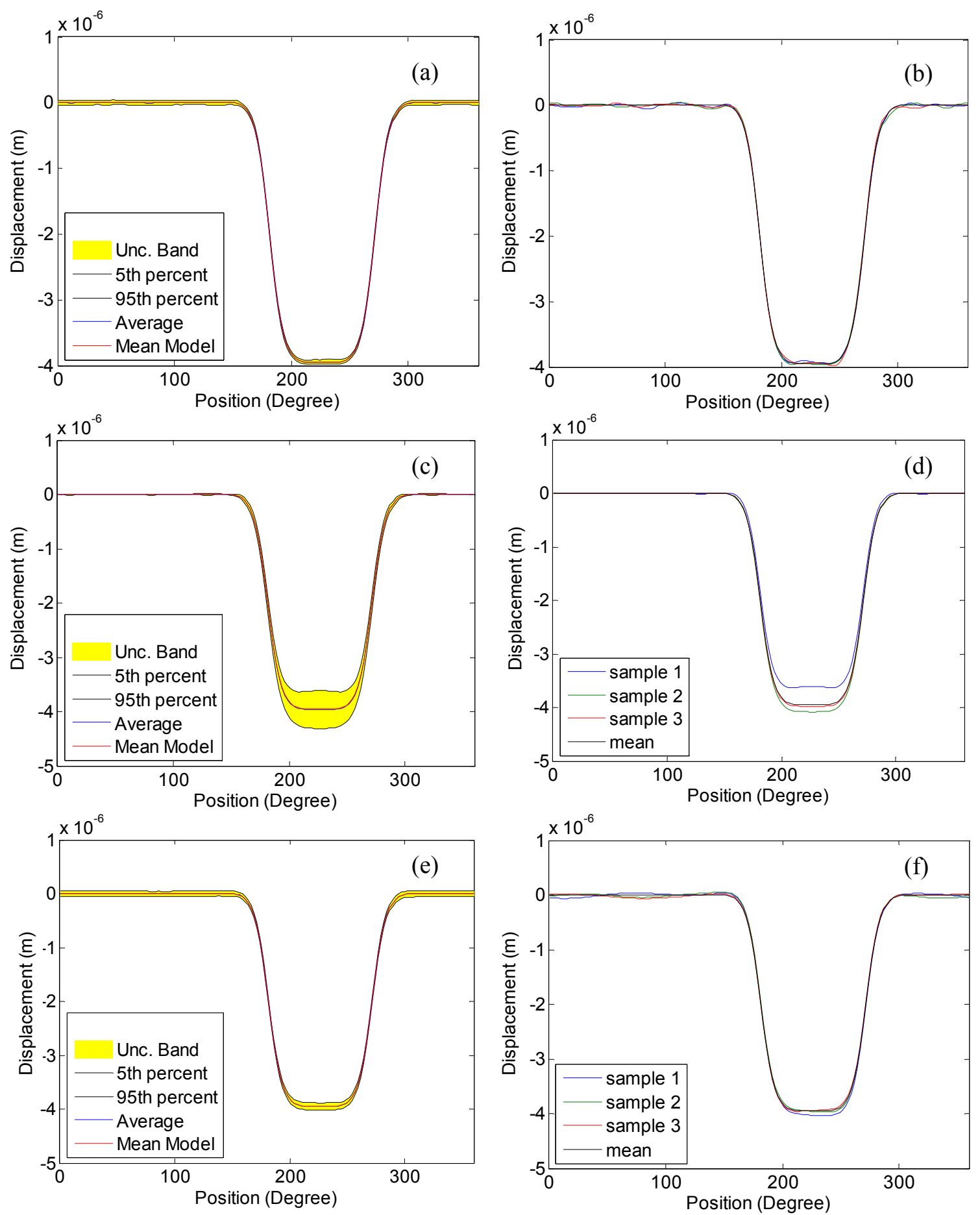

Figure 6: Static transverse displacement at the periphery of the mean annulus (in red) and results from the stochastic reduced order model: (a),(c),(e) 5th-95th percentile uncertainty band (in yellow), (b),(d),(f) 3 samples. $\left(\delta_{G}, \delta_{1}, \delta_{L}\right)=(\mathrm{a}),(\mathrm{b})(0.1,0,0)$; (c),(d) $(0,0.05,0)$;

(e),(f) $(0,0,0.02)$. 
From Figs 6 (a),(b), corresponding to $\delta_{G} \neq 0$, it is observed that the randomization of $\bar{K}_{G}$ only leads to a small, rather constant, global uncertainty band consistently with the above discussions. Next, consider Figs 6 (c),(d) corresponding to $\delta_{1} \neq 0$ and which leads to a very localized large variability of the response confirming that $K_{L}$ does indeed control the localized behavior. Finally, shown in Figs 6 (e),(f) are the results corresponding to $\delta_{L} \neq 0$ which are very similar to those shown in Figs 6 (a),(b) suggesting tentatively that it is sufficient to only consider the two parameter model defined by $\delta_{G}$ and $\delta_{1}$, i.e., with

$$
K_{L}=H_{1}^{2} \bar{K}_{L} \text {. }
$$

Shown in Fig. 7 are the uncertainty band (Fig. 7(a)) and 3 samples of the response (Fig. 7(b)) corresponding to the combined case of all three parameters nonzero. It is seen that their effects approximately superpose, creating a thin uncertainty band away from the localization region but a much more significant one within it and samples that exhibit the localization as was desired.
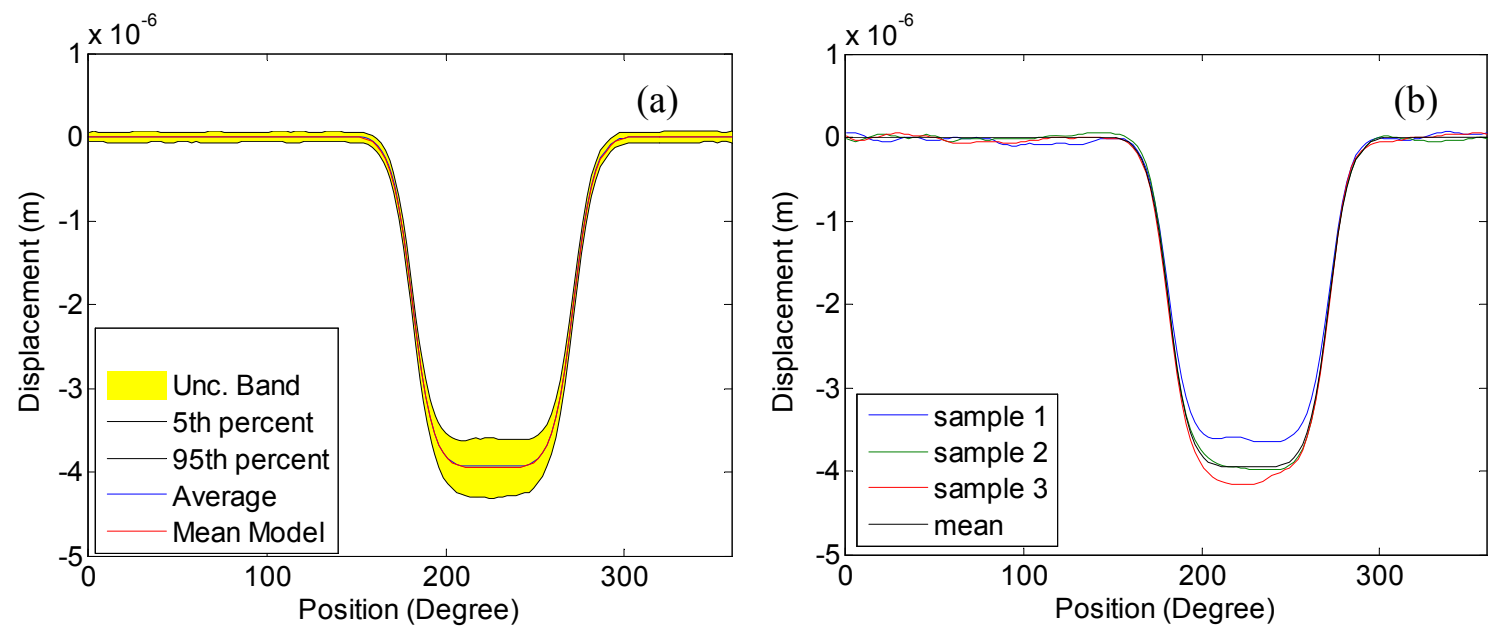

Figure 7: Static transverse displacement at the periphery of the mean annulus (in red) and results from the stochastic reduced order model: (a) 5th-95th percentile uncertainty band (in yellow), (b) 3 samples. $\left(\delta_{G}, \delta_{1}, \delta_{L}\right)=(0.1,0.05,0.02)$.

\section{SUMMARY}

This investigation focused on the development of a stochastic model of uncertainty in structures exhibiting a localized static response in the neighborhood of the excitation. More specifically, this modeling is carried out on a reduced order model of the response by relying on the maximum entropy framework. It is found that the "standard" nonparametric method leads to a local response of the simulated uncertain structures when the lowest eigenvalues of the mean structure's stiffness matrix are well separated. However, when these eigenvalues are closely spaced, this method leads to a globalization of the response which seems consistent with the maximization of the entropy. For these situations, a novel model is proposed which is based on a split of the mean structure's stiffness matrix into a component that promotes the local response and one that induces a more global behavior. Then, separate, maximum entropy based, stochastic models of these two components are carried out before they are recombined to form the stiffness matrix of the uncertain structure. This process provides control over the separation of the eigenvalues of the uncertain stiffness matrices and thus permits the 
occurrence of localization. The above findings are confirmed on an annulus clamped at its inner radius and free at the outer one and the localization of its uncertain response obtained with the proposed model is confirmed.

\section{ACKNOWLEDGEMENTS}

The financial support of this work by the Air Force Multi University Research Initiative contract FA9550-15-1-0038 with Dr. Jean-Luc Cambier as Technical Monitor is gratefully acknowledged.

\section{REFERENCES}

[1] C. Soize, A nonparametric model of random uncertainties on reduced matrix model in structural dynamics. Probabilistic Engineering Mechanics, 15, 277-294, 2000.

[2] C. Soize, Stochastic Models of Uncertainties in Computational Mechanics. American Society of Civil Engineers (ASCE), 2012.

[3] J.-F. Durand, C. Soize, C., L. Gagliardini, Structural-acoustic modeling of automotive vehicles in presence of uncertainties and experimental identification and validation. Journal of the Acoustical Society of America, 124, 1513-1525, 2008.

[4] R. Ohayon, C. Soize, Advanced Computational Vibroacoustics - Reduced-Order Models and Uncertainty Quantification, Cambridge University Press, New York, 2014.

[5] R. Murthy, M.P. Mignolet, A. El-Shafei, Nonparametric stochastic modeling of structural uncertainty in rotordynamic systems: Part I - Formulation. Journal of Engineering for Gas Turbines and Power, 132, 2010.

[6] R. Murthy, M.P. Mignolet, A. El-Shafei, Nonparametric stochastic modeling of structural uncertainty in rotordynamic systems: Part II - Applications. Journal of Engineering for Gas Turbines and Power, 132, 2010.

[7] R. Murthy, J. Tomei, X.Q. Wang, M.P. Mignolet, A. El-Shafei, Nonparametric stochastic modeling of structural uncertainty in rotordynamics: unbalance and balancing aspects. Journal of Engineering for Gas Turbines and Power, 136, 2014.

[8] M.P. Mignolet, C. Soize, Stochastic reduced order models for uncertain geometrically nonlinear dynamical systems. Computer Methods in Applied Mechanics and Engineering, 197, 3951-3963, 2008.

[9] E. Capiez-Lernout, C. Soize, M.P. Mignolet, Post-buckling nonlinear static and dynamical analyses of uncertain cylindrical shells and experimental validation. Computer Methods in Applied Mechanics and Engineering, 271, 210-230, 2014.

[10] P. Song, X.Q. Wang, A. Matney, R. Murthy, M.P. Mignolet, Nonlinear geometric thermoelastic response of structures with uncertain thermal and structural properties. Proceedings of the AIAA Science and Technology Forum and Exposition (SciTech2017), AIAA Paper AIAA 2017-0181, 2017.

[11] C. Soize, I.E. Poloskov, Time-domain formulation in computational dynamics for linear viscoelastic media with model uncertainties and stochastic excitation. Computers and Mathematics with Applications, 64, 3594-3612, 2012.

[12] A. Batou, C. Soize, Rigid multibody system dynamics with uncertain rigid bodies. Multibody System Dynamics, 27, 285-319, 2012. 
[13] LA. Richter, M.P. Mignolet, Stochastic modeling of uncertain mass characteristics in rigid body dynamics. Mechanical Systems and Signal Processing, 87, 43-53, 2017.

[14] B. Kiflu, Analysis of Mistuned Bladed Disks by a Reduced Order Technique: Validation, Identification, and Stochastic Modeling, M.S. Thesis, Arizona State University, May 2005. 